\title{
THE TIDAL RADII OF GLOBULAR CLUSTERS IN M31
}

\author{
J. G. COHEN \\ California Institute of Technology, Palomar Observatory, Mail Code 105-24, Pasadena, California 91125 \\ K. C. Freeman \\ Mount Stromlo and Siding Spring Observatory and Australian National University, Private Bag, Woden, PO, 2606 Canberra, ACT, Australia \\ Received 11 July 1990; revised 16 October 1990
}

\begin{abstract}
We have determined tidal radii for 30 globular clusters in the halo of M31 from deep direct images. Although the uncertainty of each individual measurement is large, in the mean the tidal radii suggest that the M31 globular clusters have orbital eccentricities and mass-to-light ratios that are very similar to those of galactic globular clusters.
\end{abstract}

\section{INTRODUCTION}

The structure of globular clusters is determined by internal dynamics and external forces such as the galactic tidal field. To first order, galactic globular clusters are spherical: White \& Shawl (1987) found the mean deprojected ellipticity is $0.12 \pm 0.01$. The radial surface-brightness profile of most clusters is well represented by the simple two parameter King $(1962,1966)$ models, although more elaborate multimass models provide a better fit to the profiles of some clusters (e.g., M3: Gunn \& Griffin 1979; 47 Tuc: Meylan 1989).

The tidal radius $r_{\mathrm{t}}$ of the King models represents the radius at which the cluster is truncated by the tidal field of the Galaxy. The relationship between the observed tidal radius and the location of the inner Lagrange point, as the cluster orbits in the galactic potential field, has been controversial (e.g., Innanen et al. 1983). However, Seitzer (1985) has confirmed with $N$-body calculations that the tidal radius for clusters in eccentric orbits is close to the radius of the instantaneous Lagrange point at perigalacticon, where the tidal field is maximum. (For clusters with small perigalactic radii $R_{\mathrm{p}}$ in the solid-body region of the galactic rotation curve, we note that the maximum in the tidal field may occur at $R>R_{\mathrm{n}}$.)

In a spherical logarithmic potential, the tidal radius is then given by

$$
\frac{r_{\mathrm{t}}}{\mathscr{M}_{\mathrm{cl}}^{1 / 3}}=\left(\frac{R_{\mathrm{p}}}{V}\right)^{2 / 3}\left(\frac{G}{2 g(e)}\right)^{1 / 3},
$$

where $V$ is the amplitude of the flat galactic rotation curve, $\mathscr{M}_{\mathrm{cl}}$ is the cluster mass, and $g(e)$ is a slowly varying function of the orbital eccentricity, $e=\left(R_{\mathrm{a}}-R_{\mathrm{p}}\right) /\left(R_{\mathrm{a}}+R_{\mathrm{p}}\right): R_{\mathrm{a}}$ is the orbital apogalactic radius and $g(0)=1$.

From Eq. (1), the quantity $r_{\mathrm{t}} / \mathscr{M}_{\mathrm{cl}}^{1 / 3}$ is a measure of the perigalactic radius $R_{\mathrm{p}}$, scaled by the amplitude of the galactic rotation curve. If $r_{\mathrm{t}}$ could be measured precisely for a sample of globular clusters, one could in principle combine this information with radial-velocity data to derive constraints on the mean-orbital properties of the cluster system, as was attempted for the galactic globular clusters by Peterson (1974), Seitzer (1983), and Innanen et al. (1983). It would be particularly interesting to have a sample of $r_{t}$ values for globular clusters in at least one other disk galaxy. This would provide a fairly direct comparison of the meanorbital properties of the clusters in the two galaxies, and would give some useful guidance about the more general question of the formation of globular cluster systems in disk galaxies.

Surface-brightness profiles are now available for many clusters in the LMC. However, the LMC clusters are not really suitable for this kind of comparative study because, unlike the galactic clusters, most of them belong to the disk of their parent galaxy (Freeman et al. 1983). Also, the LMC clusters cover a wide range in age. Many of the younger clusters are too young to have reached a quasi-steady state in the LMC's tidal field. Elson et al. (1987, 1989) and Lupton et al. (1989) have found examples of young LMC clusters that do not yet show the expected tidal cutoff in their surface-brightness profile. Furthermore, some of the LMC clusters are highly elliptical (Fall \& Frenk 1985, and references therein), unlike the galactic clusters.

The globular cluster system of M31 is more suitable for a comparative study, because the M31 clusters are known to be old (Frogel et al. 1980) and are close analogs of the galactic globular clusters. This view was challenged by Burstein $e t$ al. (1984), but the large sample of data of Brodie \& Huchra (1990) fail to substantiate their claim. Spassova et al. (1988) and Lupton (1989) have measured the ellipticities of a small sample of cluster in M31 and find that they are indistinguishable in the mean from those of the galactic globular clusters.

In this paper we present measurements of tidal radii for 30 globular clusters in M31. Section 2 describes the observations and the fitting procedure used to find $r_{\mathrm{t}}$. In the following section we discuss the inferred properties of the galactic and M31 cluster systems. The final section summarizes our results.

\section{OBSERVATIONS AND DETERMINATION OF $r_{\text {}}$}

\subsection{Observational Data}

Images were obtained on nights of good seeing in August 1984 of selected fields in M31 using the Four-Shooter (Gunn et al. 1984) at the Cassegrain focus of the $5 \mathrm{~m}$ telescope on Palomar Mountain. The scale is $0.34 \mathrm{arcsec}_{\mathrm{pixel}}^{-1}$, and there are four separate Texas Instruments $800 \times 800$ CCDs. The fields were selected starting at the nucleus of the galaxy and moving out along the minor axis to NGC 205, then they were centered on suspected globular clusters beyond NGC 205 from the catalog of Sargent, et al. (1977). The typical seeing was just under 1 ". Guided exposures of various lengths were taken through the $g$ and $r$ filters of the ThuanGunn (1976) system. The exposure lengths were chosen so 
that the central region of the cluster was not saturated in the shortest exposure, while the longest exposure had the sky level at about one quarter of the available dynamic range. The maximum exposure time was $800 \mathrm{~s}$. In a typical field, three useful exposures were obtained. The nights were photometric, and standard stars were observed to provide a calibration of the magnitude scale.

The frames were erase subtracted and flattened in the usual way. The clusters were identified using the charts in Sargent et al. (1977). K5, K6, K7, K145, and K320 were found to be background galaxies.

Radial profiles were measured by centroiding the image, then assembling each pixel in a plot of intensity against distance from the centroid of the image to the pixel center. The intensities were averaged over 1 pixel intervals in radius to define the radial spatial intensity profile. To measure the point-spread function, pointlike images were chosen in each frame on the same CCD detector of the Four-Shooter that were as free of contamination by adjacent stars and as clear of the background from the field of M31 as possible. The radial profiles of four point sources in each chip were measured and averaged. The profiles were calculated out to 25 pixels ( $\left.8.3^{\prime \prime}\right)$ from the centroid of the images. The outermost pixels were used to define the background level for each image, and the surface-brightness profile with the background removed was calculated. When the central pixels of a globular were saturated, the profile was extended inward using the spatial profile from a shorter exposure.

In the best cases, the spatial profiles for the clusters extend 5 " out from the center, reaching a level of about 0.004 times the peak intensity, and about $10 \%$ of the sky background level. Beyond this level, the partial resolution into stars of the background light from the field of M31 and the strong spatial variation of the background light made accurate measurements impossible. All the clusters included in our sample were spatially resolved, and the surface-brightness profiles agree well from frame to frame. Typical examples of the profiles of the M31 globular clusters are shown in Fig. 1.

\subsection{Determination of $r_{\mathrm{t}}$}

Finding a suitable algorithm to determine $r_{\mathrm{t}}$ was not easy. Let $I_{i}$ be the globular cluster profile at pixel $i$ from the centroid, and $K_{i}\left(r_{\mathrm{t}}, r_{\mathrm{c}}\right)$ be the King profile for $r_{\mathrm{t}}$ and core radius $r_{\mathrm{c}}$, convolved in two dimensions with the point-spread function. The profiles from King (1962) were used in preference to those of King (1966) because they are analytical functions and are computationally more convenient, as well as for consistency with previous work in this field (Battistini et al. 1982; Crampton et al. 1985). The brute force technique of minimizing $\chi^{2}$, where

$$
\chi^{2}=\sum\left(I_{i}-K_{i}\right)^{2} / \sigma_{i}^{2}
$$

was tried, however the appropriate definition of $\sigma$ is elusive. Lupton (1989) found that a reasonable value for $\sigma^{2}$ for pixels representing the sky at a point $2.2 \mathrm{kpc}$ from the major axis of M31 was $2.7 \sigma_{\mathrm{p}}^{2}$, where $\sigma_{\mathrm{p}}$ is that expected from Poisson statistics ignoring fluctuations in the background. The extra factor of 2.7 is presumably the effect on the statistics of the partial resolution into stars and the spatial variations in the galaxy background. But even the use of $\sigma^{2}=2.7 \sigma_{\mathrm{p}}^{2} /$ $\left(2 \pi R_{i}\right)$, where $R_{i}$ is the radius, and the factor of $2 \pi R_{i}$ takes into account the fact that further out in the profile there are more pixels in the annulus averaged together to determine $I_{i}$, is inadequate. The errors induced by the uncertainties in the point-spread function are not included, and those surely affect the innermost points more seriously than the outer points in the globular cluster surface-brightness profile. Furthermore, excessive weight cannot be placed on the outermost points, as any uncertainty in the background determination has a serious affect there.

The compromise used to determine the values of $r_{\mathrm{t}}$ is to use only the region in the normalized surface-brightness profile between 1.3 and 2.7 arcsec (corresponding to pixels 4-8) from the centroid of the image of each globular cluster. In this region, the globular cluster image profiles were smoothed with a flexible spline, and then two parameters were calculated from each profile, $J_{8}=\log \left(I_{8} / I_{1}\right)$, and a curvature parameter, $C=J_{6}-\left(J_{8}+J_{4}\right) / 2$. Typical values of $J_{8}$ range from -1.4 to -2.1 .

The stellar point-spread function fluctuated slightly from exposure to exposure, and we were unable to find a simple analytic representation. Instead, the mean stellar psf $S^{\mathrm{m}}$ for all the exposures was obtained, and it was found that for any particular exposure, the psf could be well represented by $\log \left(S_{i}\right)=k \log \left(S_{i}^{\mathrm{m}}\right)$, where $i$ is the distance in pixels from the centroid of the profile. The $k$ values were determined for each exposure, and are a measure of the width of the psf, with smaller images having larger $k$ values. The values of $k$ varied from 0.88 to 1.16 over the complete set of exposures.

For each value of $k$, a grid of King models was constructed with $r_{\mathrm{t}}$ varying over the range from 10 to $60 \mathrm{pc}$, and concentration $\left(r_{\mathrm{t}} / r_{\mathrm{c}}\right)$ varying from 10 to 100 , and convolved in two dimensions with the appropriate psf. Then contours of $J_{8}$ and of $C$ were plotted, and the observed values used to obtain $r_{\mathrm{t}}$. An example of a set of contours for $k=1.00$ is given in Fig. 2.

Table 1 lists the observed parameters of the M31 globular cluster image profiles, specifically the mean values of $k, J_{8}$, and $C$ averaged over the set of frames for each cluster. Table 2 lists the derived values of $r_{\mathrm{t}}$, which are the averages over the set of images for each cluster, for 30 globular clusters in M31. A true distance modulus of $24.26 \mathrm{mag}$ (Welch et al. 1986) is used. Also included are the photometric data (for an aperture diameter of $7.5 \mathrm{arcsec}$ ), and the projected distance $R_{\mathrm{GC}}$ of each cluster from the center of M31. The core radii are too uncertain, and are not given. Instead, we give the half light radius $r_{\mathrm{e}}$ calculated from the analytical function given by King (1962) from the deduced best-fit values of $r_{\mathrm{t}}$ and $r_{\mathrm{c}}$. Note that when the photometric data are omitted, it was because the central pixels of all frames taken through the $r$ filter of a particular cluster were saturated. (The shortest $g$ images were never saturated.)

\subsection{Error Estimates for $r_{\mathrm{t}}$}

The values of $r_{\mathrm{t}}$ are uncertain because of uncertainties in the surface-brightness profiles for the globular clusters and in the stellar point-spread function applied to each frame. To illustrate the nature of the former, we plot $\chi^{2}$ for a particular frame of the M31 globular cluster $\mathrm{K} 312$, where we use $\sigma_{i}^{2}=2.7 \sigma_{\mathrm{p}, i}^{2} /\left(2 \pi R_{i}\right)$, and all calculations are in electrons. The $r_{\mathrm{t}}$ values estimated from the minimum $\chi^{2}$ are within $10 \%$ of those from the $\left(J_{8}, C\right)$ fitting procedure. Thus the spline smoothing applied before the $\left(J_{8}, C\right)$ procedure does not introduce significant errors.

The value of $\chi_{v}^{2}$ per degree of freedom as a function of $r_{\mathrm{t}}$ 


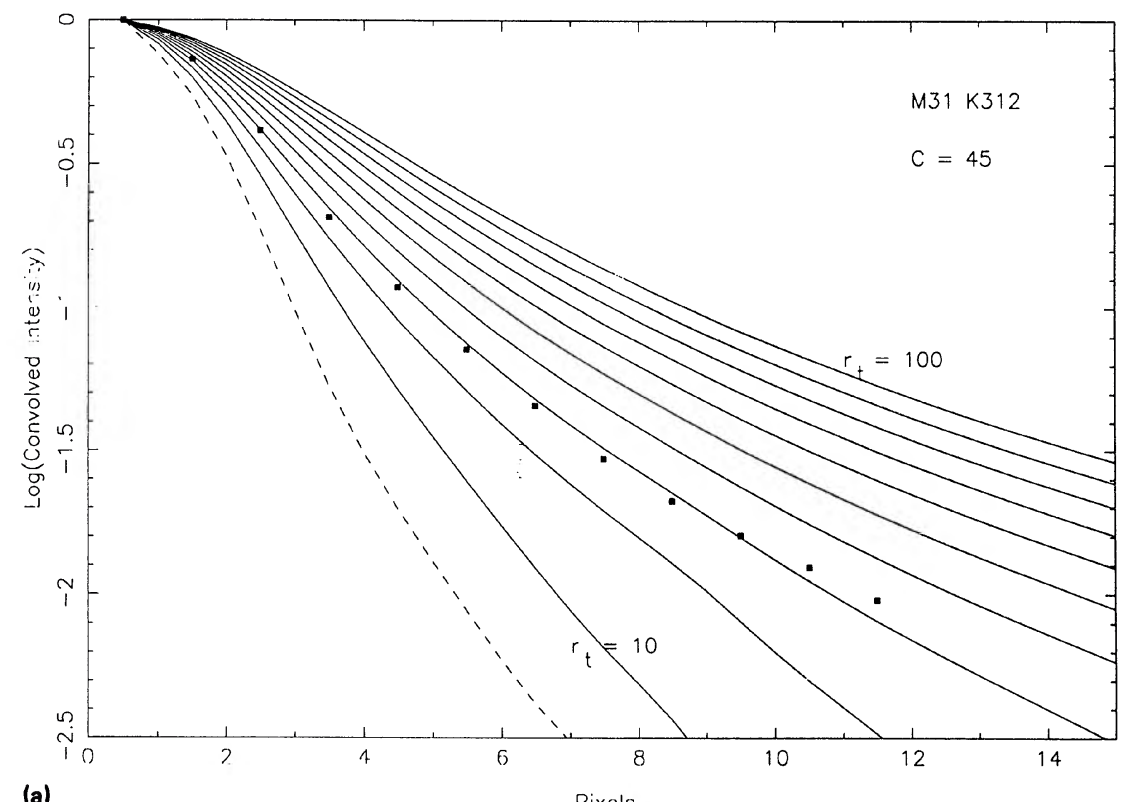

FIG. 1. (a) The observed spatial profile of the cluster K312 in M31 is indicated by the filled symbols. The seeing is shown as the dashed line. The predicted profiles for a fixed $c=r_{1} / r_{\mathrm{c}}$ of 45 for $r_{1}$ from 10 to 100 pixels in steps of 10 pixels are shown by the

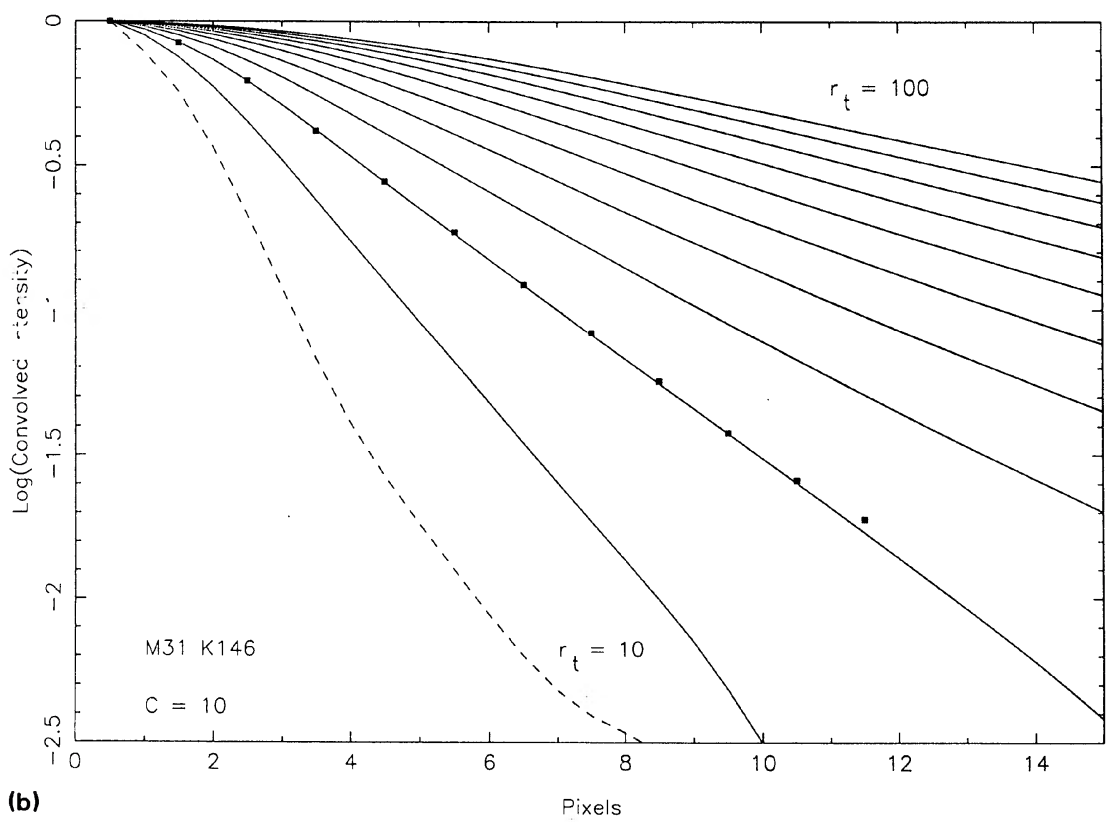
solid curves. (b) The same as (a) for the cluster K146 in M31. Note that the seeing is somewhat poorer than that in (a).

(b)

$$
\text { Pixels }
$$




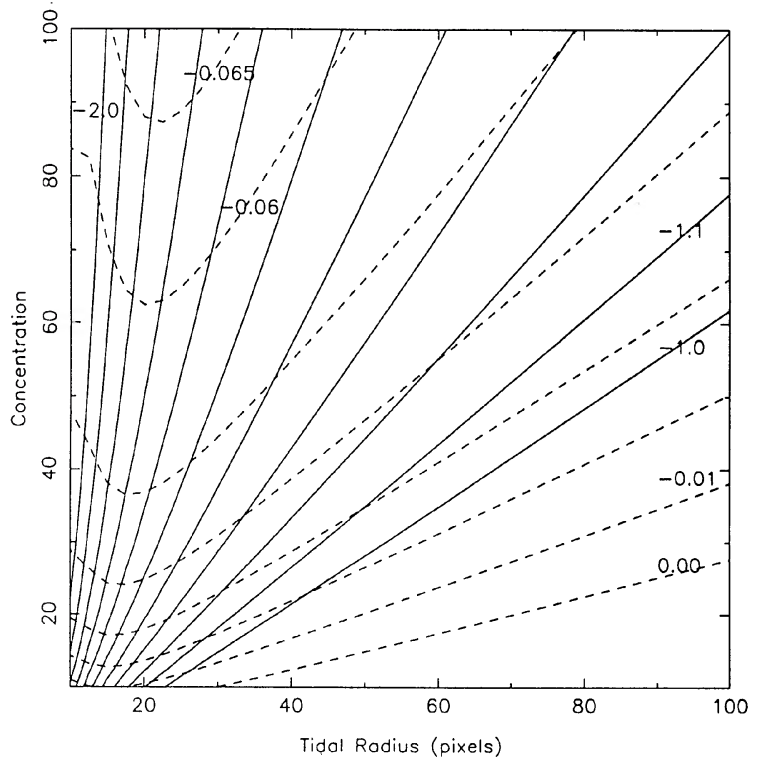

FIG. 2. A grid of King models has been calculated for varying tidal radii and concentration $\left(r_{1} / r_{\mathrm{c}}\right)$ with the seeing parameter $k=1$. Contours of constant $J_{8}$ (solid lines) ranging from -2.0 to $-1.0 \mathrm{dex}$ in steps of 0.10 dex are shown. The dashed lines are contours of constant curvature $C$.
TABLE 2. Properties of the M31 globular clusters.

\begin{tabular}{lccrcc}
\hline \hline ID & $\begin{array}{c}r \\
(\mathrm{mag})\end{array}$ & $\begin{array}{c}g-r \\
(\mathrm{mag})\end{array}$ & $\begin{array}{c}R_{\mathrm{gc}} \\
(\mathrm{kpc})\end{array}$ & $\begin{array}{c}r_{\mathrm{t}} \\
(\mathrm{pc})\end{array}$ & $\begin{array}{c}r_{\mathrm{e}} \\
(\mathrm{pc})\end{array}$ \\
\hline vdB5 & & & 0.5 & 23 & 2.1 \\
K25 & 18.71 & 0.91 & 10.7 & 48 & 3.1 \\
K108 & 15.62 & 0.44 & 4.3 & 63 & 3.5 \\
K109 & 17.56 & 0.43 & 6.5 & 15 & 2.6 \\
K111 & 17.21 & 0.40 & 5.7 & 19 & 3.7 \\
K122 & & & 3.1 & 39 & 2.6 \\
K124 & 15.23 & 0.71 & 3.1 & 21 & 2.2 \\
K132 & 18.30 & 0.02 & 2.5 & 20 & 4.0 \\
K135 & 16.56 & 0.38 & 5.7 & 23 & 2.7 \\
K143 & 16.62 & 0.44 & 11.8 & 22 & 1.5 \\
K146 & 16.90 & 0.42 & 6.0 & 21 & 4.0 \\
K151 & 17.99 & 0.00 & 1.5 & 38 & 2.6 \\
K155 & 16.49 & 0.51 & 1.4 & 28 & 2.7 \\
K159 & 16.48 & 0.53 & 2.0 & 18 & 3.0 \\
K165 & & & 0.7 & 20 & 2.5 \\
K169 & & & 0.9 & 18 & 1.7 \\
K174 & & & 0.6 & 31 & 2.3 \\
K177 & & & 0.5 & 11 & 1.1 \\
K180 & 16.79 & 0.39 & 9.3 & 19 & 2.6 \\
K184 & 16.6 & 0.6 & 15 & 1.8 \\
K185 & & & 0.2 & 20 & 2.9 \\
K189 & & & 0.3 & 13 & 2.2 \\
K194 & 16.68 & 0.30 & 0.8 & 23 & 2.2 \\
K198 & 15.76 & 0.39 & 0.9 & 18 & 1.7 \\
K200 & & & 0.8 & 12 & 1.3 \\
K202 & 17.37 & 0.35 & 9.3 & 27 & 4.3 \\
K207 & 15.86 & 0.40 & 1.1 & 20 & 2.3 \\
K208 & 16.62 & 0.50 & 1.1 & 22 & 2.7 \\
K308 & 17.30 & 0.61 & 10.3 & 41 & 3.8 \\
K312 & 15.95 & 0.69 & 10.0 & 34 & 2.9 \\
\hline \hline
\end{tabular}

and $r_{\mathrm{c}}$, using the full surface-brightness profile for this cluster, is shown in Fig. 3. The contours increase by a factor of 2 per level. It is immediately obvious from this figure that the uncertainties in $r_{\mathrm{c}}$ are much larger than those of $r_{\mathrm{t}}$, and no reliable measurements of the former parameter can be obtained from the present data.

TABLE 1. Observed parameters of the M31 globular cluster image profiles.

\begin{tabular}{|c|c|c|c|}
\hline ID & $\langle k\rangle$ & $\begin{array}{c}\left\langle J_{8}\right\rangle \\
(\operatorname{dex})\end{array}$ & $\langle C\rangle$ \\
\hline $\begin{array}{l}\text { vdB5 } \\
\text { K25 } \\
\text { K108 } \\
\text { K109 } \\
\text { K111 } \\
\text { K122 } \\
\text { K124 } \\
\text { K132 } \\
\text { K135 } \\
\text { K143 } \\
\text { K146 } \\
\text { K151 } \\
\text { K155 } \\
\text { K159 } \\
\text { K165 } \\
\text { K169 } \\
\text { K174 } \\
\text { K177 } \\
\text { K180 } \\
\text { K184 } \\
\text { K185 } \\
\text { K189 } \\
\text { K194 } \\
\text { K198 } \\
\text { K200 } \\
\text { K202 } \\
\text { K207 } \\
\text { K208 } \\
\text { K308 } \\
\text { K312 }\end{array}$ & $\begin{array}{l}1.01 \\
1.09 \\
1.02 \\
1.04 \\
1.08 \\
0.98 \\
0.97 \\
0.98 \\
1.02 \\
0.95 \\
1.08 \\
0.97 \\
0.97 \\
1.00 \\
1.05 \\
1.00 \\
1.02 \\
1.01 \\
0.94 \\
1.01 \\
1.00 \\
1.02 \\
1.03 \\
1.03 \\
1.03 \\
1.01 \\
1.03 \\
0.98 \\
1.11 \\
1.04\end{array}$ & $\begin{array}{l}-1.74 \\
-1.46 \\
-1.38 \\
-1.79 \\
-1.26 \\
-1.53 \\
-1.59 \\
-1.12 \\
-1.37 \\
-1.52 \\
-1.10 \\
-1.52 \\
-1.45 \\
-1.57 \\
-1.64 \\
-1.79 \\
-1.58 \\
-2.00 \\
-1.42 \\
-1.76 \\
-1.52 \\
-1.66 \\
-1.63 \\
-1.89 \\
-2.05 \\
-0.96 \\
-1.62 \\
-1.37 \\
-1.29 \\
-1.51\end{array}$ & $\begin{array}{l}-0.060 \\
-0.058 \\
-0.055 \\
-0.027 \\
+0.015 \\
-0.060 \\
-0.043 \\
+0.028 \\
-0.033 \\
-0.045 \\
+0.005 \\
-0.059 \\
-0.047 \\
-0.033 \\
-0.045 \\
-0.057 \\
-0.060 \\
-0.040 \\
-0.030 \\
-0.030 \\
-0.040 \\
-0.010 \\
-0.052 \\
-0.060 \\
-0.042 \\
-0.005 \\
-0.045 \\
-0.030 \\
-0.038 \\
-0.055\end{array}$ \\
\hline
\end{tabular}

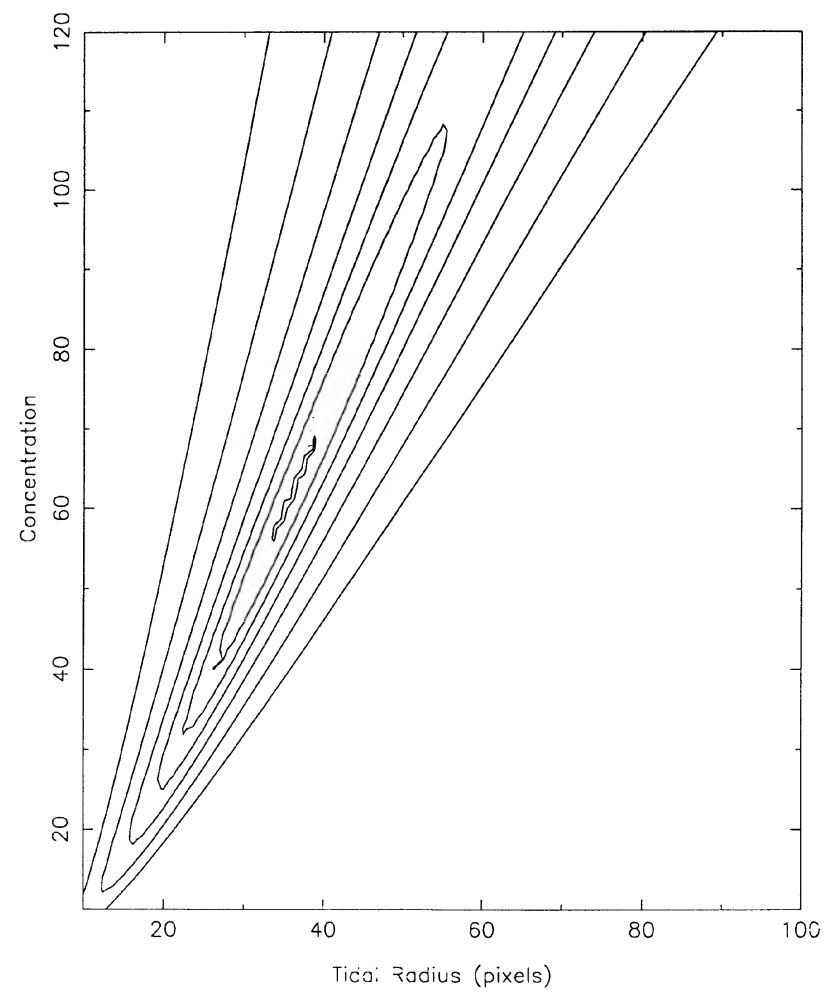

FIG. 3. Contours of constant $\chi^{2}$. ( $\chi^{2}$ per degree of freedom) are shown for fitting King models as a function of $r_{\mathrm{t}}$ and concentration $\left(r_{\mathrm{t}} / r_{\mathrm{c}}\right)$ to the observed profile of the globular cluster K 312 . The value of $\chi_{\text {r }}^{2}$ increases by a factor of 2 between each contour. 
The minimum value of $\chi_{v}^{2}$ obtained over the entire parameter space of $r_{\mathrm{t}}$ and $r_{\mathrm{c}}$ is significantly larger than expected, which suggests that either the model is not completely appropriate or the choice we have made for representing the uncertainties of each point in the profile is a serious underestimate. The same underestimate of the errors when represented as purely Poisson statistics can be seen upon examination of the full radial intensity profile including all points prior to azimuthal averaging. The spread at any intensity level within the raw data is larger than expected. This could be due to a small ellipticity in the globular cluster image or to stochastic fluctuations of the number of bright giants within each pixel a la Tonry \& Schneider (1988).

Our error estimate for $r_{\mathrm{t}}$ is based on allowing the minimum achieved $\chi_{v}^{2}$ to double in value. This implies that the errors in $r_{\mathrm{t}}$ for each measurement are $+70 \%,-15 \%$. Similar conclusions about the magnitude of the errors can be reached by considering the likely magnitude of the errors in $J_{8}$ and in $C$, and examining Fig. $1 . J_{8}$ is relatively well determined, with an uncertainty of less than 0.05 dex. But the curvature parameter $C$ is poorly measured, and this produces large uncertainties in $r_{\mathrm{t}}$, and even larger ones in $r_{\mathrm{c}}$.

The repeatability of our estimates of $r_{\mathrm{t}}$ and $r_{\mathrm{c}}$, from frame to frame of a given cluster, is much better than the errors quoted above: the range is only about $\pm 10 \%$. However, these frame-to-frame variations include only the uncertainties in the point-spread function and the photometry; they do not include systematic errors due to the background fluctuations, which affect all profiles of a particular cluster in a similar way.

Realistic errors in the psf do not induce large errors in $r_{\mathrm{t}}$. For example, $10 \%$ changes in the $k$ values for the psf change the $r_{\mathrm{t}}$ estimates by less than $15 \%$. This is to be expected, because seeing was good and the cluster profiles are relatively extended.

\subsection{Comparison with Previous Studies}

Two previous studies of the structural parameters of the M31 globular clusters present estimates of core radii, which were derived indirectly from the full width of the cluster profile at quarter maximum using a transformation from FWQM to $r_{\mathrm{c}}$ calculated from King (1962) models. Our core radii agree well with those of Battistini et al. (1982); there is no significant systematic difference between our two sets of values, and the standard deviation of $\left[r_{\mathrm{c}}\right.$ (this paper) $-r_{\mathrm{c}}$ (Battistini et al.)] is about $50 \%$ of $r_{\mathrm{c}}$. This good agreement may seem at variance with our earlier comments concerning the large uncertainties in our $r_{\mathrm{c}}$ estimates. However, both sets of $r_{\mathrm{c}}$ values are similarly affected by fluctuations in the background, and we believe that the true errors in $r_{\mathrm{c}}$ are larger than suggested by this comparison of the two data sets. Crampton et al.'s (1985) values of $r_{\mathrm{c}}$ are systematically about $50 \%$ larger than our values or those of Battistini et al. (1982).

Seeing deconvolution was carried out for a sample of only six clusters in M31 by Bendinelli et al. (1990). There is no overlap with our sample, and the effort of that work is directed at determining the presence of post-core collapse clusters rather than at measuring $r_{\mathrm{t}}$.

\section{INFERENCES FROM THE MEASURED TIDAL RADII}

We wish to determine whether the measured tidal radii derived above are consistent with the values we would expect if the M31 globular clusters were exact analogs in stellar content and orbital properties of the galactic globular clusters. For the latter, the mass-to-light ratios are approximately constant at $\mathscr{M} / L_{V} \approx 2$ (Illingworth 1976; Peterson \& Latham 1986). Since the individual values of $r_{\mathrm{t}}$ are very uncertain, we proceed by finding the average values of the tidal radius for the sample of $\mathrm{M} 31$ globular clusters within two ranges of projected galactocentric distance: $R_{\mathrm{GC}}<4$ $\mathrm{kpc}$, and $4<R_{\mathrm{GC}}<16 \mathrm{kpc}$. To remove the dependence of $r_{\mathrm{t}}$ on the cluster mass, we assume that $\mathscr{M} / L_{V}=2.0$, and use the observed integrated luminosities to calculate average values of $P=r_{\mathrm{t}} / \mathscr{M}_{\mathrm{cl}}^{1 / 3}$ for our sample of globular clusters in M31. These values are given in Table 3 and plotted in Fig. 4(b).

Note that our sample of M31 globular clusters is magnitude limited, and has a median $V$ of about $16.7 \mathrm{mag}$ ( $\mathscr{M}_{V} \approx-7.9 \mathrm{mag}$ ). Thus we confine the sample of galactic globular clusters taken from Webbink's (1985) compilation to those with $\mathscr{M}_{V}<-7.1 \mathrm{mag}$ (i.e., the brighter half of the distribution). The projected distances in the plane defined by $l^{\text {II }}=0^{\circ}$, with the galactic center as the origin of the coordinate system, are used to produce the values in Table 3 . The $P$ values for the galactic sample are plotted as a function of galactocentric distance in Fig. 4(a). The clusters fainter than the magnitude cutoff are shown as open circles, while the brighter ones are shown as filled symbols. Also indicated are the predictions of Eq. (1) for $R_{\mathrm{GC}}=R_{\mathrm{p}}$ and for clusters whose perigalactic distance is $R_{\mathrm{GC}} / 3$. Here we have adopted $V=220 \mathrm{~km} \mathrm{~s}^{-1}$ and $g(e)=2$, corresponding to a mean orbital eccentricity of about 0.6. A small segment of the curve for circular orbits, i.e., $R_{\mathrm{GC}}=R_{\mathrm{p}}$ and $g(e)=1$, is also shown. It is apparent that Eq. (1), with our adopted values of the parameters, gives a good description of the cluster tidal radii.

The average values of $r_{\mathrm{t}}$ for the galactic sample thus defined are also given in Table 3. However, M31 is more luminous and rotates more rapidly than the Galaxy, hence a scaling is required before we can directly compare the values of $P$ for M31 and the Galaxy. From Eq. (1), we must adjust the galactic globular cluster value of $\langle P\rangle$ by the ratio $\left(V_{\mathrm{Gal}} / V_{\mathrm{M} 31}\right)^{2 / 3}$. For $V_{\mathrm{Gal}}=220 \mathrm{~km} \mathrm{~s}^{-1}$ and $V_{\mathrm{M} 31}=265$ $\mathrm{km} \mathrm{s}^{-1}$ (Roberts 1966), this factor is 0.88. The adjusted values $\left\langle P_{c}\right\rangle$ are given in the fourth column of Table 3 . We can compare this adjustment factor with that derived for the Keplerian approximation to the galactic tidal field, in which

$$
r_{\mathrm{t}}=R_{\mathrm{p}}\left\{\mathscr{M}_{\mathrm{cl}} /\left[(3+e) \mathscr{M}_{\mathrm{g}}\right]\right\}^{1 / 3} \text {, }
$$

where $\mathscr{M}_{\mathrm{g}}$ is now the galactic mass. In this case the adjustment factor is $\left(\mathscr{M}_{\mathrm{Gal}} / \mathscr{M}_{\mathrm{M} 31}\right)^{1 / 3}$. If we adopt a difference of $0.6 \mathrm{mag}$ in integrated luminosity (Allan 1973) and assume

TABLE 3. Mean tidal radii corrected for cluster mass (and galactic rotation).

\begin{tabular}{|c|c|c|c|c|c|}
\hline \multirow[b]{2}{*}{$\begin{array}{l}R_{\mathrm{GC}} \\
(\mathrm{kpc})\end{array}$} & \multicolumn{3}{|c|}{ The Galaxy } & \multicolumn{2}{|c|}{ M31 } \\
\hline & No. & $\begin{array}{l}\left\langle r_{\mathrm{t}} / \mathscr{M}_{\mathrm{ct}}^{1 /}\right. \\
\left(\mathrm{pc} / \mathscr{M}_{\odot}^{0 .}\right.\end{array}$ & $\begin{array}{l}\left\langle r_{\mathrm{t}} / \mathscr{M}_{\mathrm{cl}}^{1 / 3}\right\rangle c \\
\left(\mathrm{pc} / \mathscr{M}_{\odot}^{0.33}\right)\end{array}$ & No. & $\begin{array}{l}\left\langle r_{\mathrm{t}} / M_{\mathrm{cl}}^{1 / 3}\right\rangle \\
\left(\mathrm{pc} / \mathscr{M}_{\odot}^{0.33}\right)\end{array}$ \\
\hline$<4$ & 27 & 0.46 & 0.41 & 19 & 0.37 \\
\hline $4<16$ & 39 & 0.73 & 0.64 & 11 & 0.60 \\
\hline
\end{tabular}



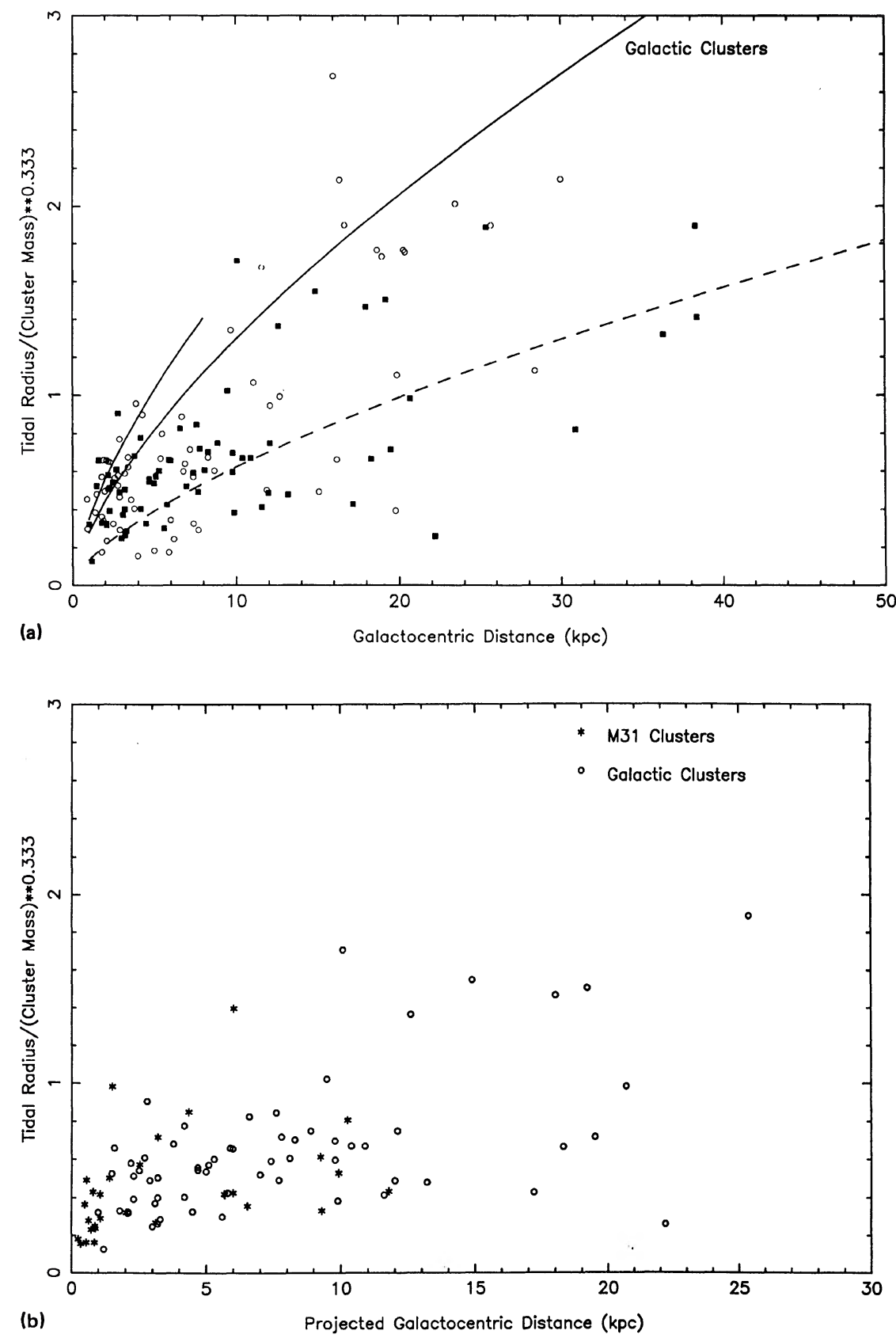

FIG. 4. (a) $r_{\mathrm{t}} / \mathscr{M}_{\mathrm{cl}}^{1 / 3}$ in units of pc (solarmass $)^{-1 / 3}$ is shown as a function of the galactocentric distance for the galactic globular clusters. The closed symbols denote clusters brighter than $\mathscr{M}_{V}=-7.1 \mathrm{mag}$ while the open symbols denote the less luminous clusters. The solid lines represent the curve of Eq. (1) assuming $R_{\mathrm{p}}=R_{\mathrm{GC}}$, with the shorter segment having $g(e)=1$ (circular orbits), while the longer segment has $g(e)=2$. The dashed curve is that of Eq. (1) for a cluster whose present galactocentric distance is $3 R_{\mathrm{p}}$ with $g(e)=2$. (b) The same as (a) using the projected galactocentric distances. The M31 clusters are denotec by stars; the galactic globular clusters with $\mathscr{M}_{V} \leqslant-7.1 \mathrm{mag}$ are plotted as open circles. 
the same $\mathscr{M} / L$ for the two galaxies, the adjustment factor would be 0.83 .

The similarity between the $\left\langle P_{\mathrm{c}}\right\rangle$ values for the brighter galactic globular clusters and $\langle P\rangle$ for our sample of globulars in M31 is impressive. From Eq. (1), this implies that the average values of $\left\langle\left[r_{p}^{2}(\mathscr{M} / L)\right] / g(e)\right\rangle$ for M31 and galactic clusters in each radial interval of Table 3 are approximately equal. The mean values of $\mathscr{M} / L$ for clusters in M31 and the Galaxy are about the same [Peterson \& Lupton (unpublished); see Peterson 1989]. It then follows that the orbital eccentricity distributions for clusters in M31 and the Galaxy are similar, to within the limits of our measurements.

\section{SUMMARY AND CONCLUSION}

It is remarkable how alike globular cluster systems are from galaxy to galaxy (see the reviews by Harris 1987, 1988 ), differing only in the absolute numbers and mean metallicities. This similarity has been confirmed yet again by our measurements of the tidal radii of 30 globular clusters in
M31. Although the individual measurements are quite uncertain, we find that in the mean the values of $r_{\mathrm{t}}$ are those we would expect if the M31 globular clusters had an orbital eccentricity distribution similar to that in the Galaxy.

It now appears that the globular clusters we see today are a pathetic remnant of some much larger initial population, only those that managed to survive such processes as tidal stripping, evaporation, repeated passages through the galactic disk, and bulge (see, for example, Aguilar, et al. 1988). If that is correct, then the properties of the cluster systems we see today primarily reflect survival statistics rather than conditions at early epochs of galaxy formation (see Fall \& Rees 1985 ). It is then interesting that the destruction mechanisms appear to operate as similarly in M31 and the Galaxy.

J.G.C. and K.C.F. are grateful to the Aspen Center for Physics for hospitality while the final draft of this paper was written. J.G.C. is grateful to the Caltech Recycling Center for financial support.
Aguilar, L., Hut, P., and Ostriker, J. P. 1988, ApJ, 335, 720

Allan, C. W. 1973, Astrophysical Quantitites (Athlone, London)

Battistini, P., Bonolo, F., Buonanno, R., Corsi, C. E., and Fusi Peci, F. 1982, A\&A, 113, 39

Bendinelli, O., Parmeggiani, G., Zavatti, F., and Djorgovski, S. 1990, AJ, 99,774

Brodie, J. P., and Huchra, J. P. 1990, ApJ (in press)

Burstein, D., Faber, S., Gaskell, M., and Krum, N. 1984, ApJ, 287, 586

Crampton, D., Cowley, A. P., Schade, D., and Chayer, P. 1985, ApJ, 288, 494

Elson, R. A. W., Fall, S. M., and Freeman, K. C. 1987, ApJ, 323, 54

Elson, R. A. W., Fall, S. M., and Freeman, K. C. 1989, ApJ, 336, 734

Fall S. M., and Frenk C. S. 1985, in Dynamics of Star Clusters, edited by J. Goodman and P. Hut (Reidel, Dorchrecht), p. 285

Fall, S. M., and Rees, M. J. 1985, ApJ, 298, 18

Freeman, K. C., Illingworth, G. D., and Oemler, A. 1983, ApJ, 272, 488

Frogel, J. A., Persson, S. E., and Cohen, J. G. 1980, ApJ, 240, 785

Gunn, J. E., Carr, M., Chang, J., Danielson, G. E. A., Lorenz, E. O., Lucinio, R., Nenow, V. E., Smith, O. J., Westphal, J. A., and Zimmerman B. A. 1984, BAAS, 16, 447

Gunn, J. E., and Griffin, R. F. 1979, AJ, 84, 752

Harris, W. E. 1987, PASP, 99, 1031

Harris, W. E. 1988, in Globular Clusters in Galaxies, edited by J. E. Grindlay and A. G. Davis Philip (Kluwer, Dordrecht), p. 237

Illingworth, G. 1976, ApJ, 204, 73

Innanen, K. A., Harris, W. E., and Webbink, R. F. 1983, AJ, 88, 338

\section{REFERENCES}

King, I. 1962, AJ, 67, 471

King, I. R. 1966, AJ, 71, 64

Lupton, R. H. 1989, AJ, 97, 1350

Lupton, R. H., Fall, S. M., Freeman, K. C., and Elson, R. A. W. 1989, ApJ, 347,201

Meylan, G. 1989, A\&A, 214, 106

Peterson, C. J. 1974, ApJ, 190, L17

Peterson, R. C. 1989, in Dynamics of Dense Stellar Systems, edited by D. Merritt, p. 161

Peterson, R. C., and Latham, D. W. 1986, ApJ, 305, 645

Roberts, M. 1966, ApJ, 144, 639

Sargent, W. L. W., Kowal, C. T., and Hartwick, F. D. A. 1977, AJ, 82, 947

Seitzer, P. 1983, Ph.D thesis, University of Virginia

Seitzer, P. 1985, in Dynamics of Star Clusters, edited by J. Goodman and P. Hut (Reidel, Dordrecht), p. 343

Spassova, N. M., Staneva, A. V., and Golev, V. K. 1988, in Globular Cluster Systems in Galaxies, edited by J. E. Grindlay and A. G. Davis Philip (Kluwer, Dordrecht) p. 569

Thuan T. X., and Gunn J. E. 1976, PASP, 88, 543

Tonry, J. L., and Schneider, D. P. 1988, AJ, 96, 807

Webbink, R. F. 1985, in Dynamics of Star Clusters, edited by J. Goodman and P. Hut (Reidel, Dordrecht), p. 541

Welch, D. L., McAlary, C. W., McLaren, R. A., and Madore, B. F. 1986, ApJ, 305, 583

White, R. E., and Shawl, S. J. 1987, ApJ, 317, 246 